\title{
Factors Affecting Antibiotic Prescribing for Acute Respiratory Infection by Emergency Physicians
}

\author{
Nasia Safdar1,2, Thomas G. Tape ${ }^{3}$, Barry C. Fox ${ }^{2}$, James E. Svenson ${ }^{4}$, Robert S. Wigton ${ }^{3}$ \\ ${ }^{1}$ William S. Middleton Memorial Veterans Hospital, Madison, USA \\ ${ }^{2}$ Division of Infectious Diseases, Department of Medicine, University of Wisconsin-Madison, Madison, USA \\ ${ }^{3}$ College of Medicine, University of Nebraska Medical Center, Omaha, USA \\ ${ }^{4}$ Section of Emergency Medicine, Department of Medicine, University of Wisconsin-Madison, Madison, USA \\ Email: ns2@medicine.wisc.edu
}

Received 10 January 2014; revised 13 February 2014; accepted 22 February 2014

Copyright (C) 2014 by authors and Scientific Research Publishing Inc.

This work is licensed under the Creative Commons Attribution International License (CC BY).

http://creativecommons.org/licenses/by/4.0/

(c) (7) Open Access

\begin{abstract}
Background: Antibiotics are frequently prescribed for upper acute respiratory tract infections (ARI) in the emergency department. To reduce inappropriate overprescribing, it is necessary to understand factors influencing physicians' decisions to prescribe antibiotics. Objective: Analyze the judgment policies of emergency physicians to determine factors predicting antibiotic use. Design: Paper case vignette study. Participants: 104 emergency physicians from Wisconsin. Measurements: We used judgment analysis to derive the policies of 104 emergency physicians from their responses to 20 case vignettes. We designed the cases such that each physician's use of clinical findings and patient factors could be inferred from the decisions they made about each case. Findings were compared to primary care practitioners (PCPs) in Colorado responding to the same paper cases to examine differences in factors influencing prescribing among the two groups. Results: The emergency physicians said they would prescribe an antibiotic (yes/no) in $51.4 \%$ of cases compared with $\mathbf{4 4 . 5 \%}$ in the Colorado study. The majority of emergency physicians gave the greatest weight to duration of illness $(51 \%)$, followed by temperature $(20 \%)$ and cough $(12 \%)$. Conclusions: These emergency physicians prescribed antibiotics more frequently than the Colorado PCPs. There was little difference in how clinical findings were weighted, suggesting this was not the source of the higher prescription rate among the emergency physicians. The considerable emphasis on duration of illness suggests that this would be a productive area for educational efforts to reduce antibiotic prescribing for ARI in the emergency department.
\end{abstract}

\section{Keywords}

\section{Antibiotic; Infection; Prescribing}




\section{Introduction}

Increase in antibiotic resistance related to antibiotic use and overuse remains a growing concern. The Centers for Disease Control has published guidelines for the appropriate use of antibiotics for upper respiratory infections and bronchitis in the hope of decreasing inappropriate use [1]-[4]. Despite consensus and recommendations that antibiotics are not necessary for the vast majority of acute respiratory infections (ARI), inappropriate prescribing of antibiotics for these conditions continues as a major public health challenge [5]. Interventions to modify antibiotic overuse, targeted at both physicians and patients have had mixed results [6] [7]. Inappropriate antibiotic use has decreased somewhat [8], but antibiotics are still inappropriately prescribed in up to half of emergency department (ED) visits for ARI [9].

Emergency providers are often cited as the worst offenders of the overuse of antibiotics [10]. Reasons given for these cited differences include: patients in the ED may be worse, may be less likely to have insurance, may lack access to good medical follow-up, and may have different expectations regarding the need and desire for antibiotics than those in primary care [11].

A better understanding of the judgment process that underlies antibiotic prescribing could improve our ability to devise appropriate strategies for reducing antimicrobial usage. We conceptualized the decision to prescribe antibiotics using a Bayesian framework. Each physician starts with an expected prior probability of prescribing antibiotics for ED patients with acute respiratory illness. For any given patient, consideration of both clinical and circumstantial patient factors then leads to a posterior probability of prescribing antibiotics. Clinical factors include signs and symptoms related to the likelihood of bacterial infection. Patient factors include a number of other reasons favoring antibiotic prescribing such as patient demand, prior experience with antibiotics, poor access to follow-up or perceived severity of illness.

Previous studies of antibiotic use for respiratory infections have predominantly focused on primary care settings and these findings may not necessarily apply to the ED setting [12]-[14]. In an earlier study using hypothetical case scenarios, primary care practitioners (PCPs) in Colorado prescribed antibiotics for ARI at twice the rate of physicians following CDC practice guidelines. The most important factor was duration of illness-circumstantial patient factors were unimportant in deciding whether to prescribe antibiotics [14]. We used these same case scenarios to identify factors that influenced antibiotic prescribing by emergency physicians and compared them to the factors used by PCPs in the earlier study.

We hypothesized that the differences in antibiotic use of ED physicians compared to primary care physicians could be accounted for by differences in how the two physician groups weight both clinical and circumstantial patient factors. We anticipated that ED physicians would give more weight to patient factors than the primary care practitioners.

\section{Methods}

To allow comparison between emergency physicians and community practitioners, we used the same methodology of judgment analysis to study Wisconsin ED physicians as that previously reported for Colorado community practitioners [14]. Judgment analysis models judgment using regression techniques to infer weights of clinical factors in hypothetical clinical vignettes based on subjects' estimates of their likelihood of prescribing antibiotics for each vignette. Because the variables in the vignettes are systematically varied according to a factorial design, subjects must make tradeoffs among factors when judging the probability they would prescribe antibiotics in a given vignette.

\subsection{Paper Case Vignettes}

We used the same 20 vignettes used in the earlier study of community practitioners' prescribing of antibiotics in ARI [14]. After reading each case, respondents were asked whether or not they would prescribe antibiotics for this patient [yes/no] and how likely it was they would prescribe antibiotics (0 - 100 scale).

The vignettes contained 9 factors, which included both clinical and patient findings (Table 1). The patient findings were those that might have affected treatment but not diagnosis, such as patient expectation for antibiotics or a pending trip or the patients' belief they were seriously ill. These factors have been found in other studies to influence prescribing decisions [13]. Each case presented the same variables in either a positive or negative form (e.g., “no cough” or "productive cough with yellow sputum”). 
Table 1. Clinical and patient factors employed in case vignettes.

\begin{tabular}{|c|c|c|}
\hline Factor & Factor absent & Factor present \\
\hline Nasal drainage & None & Colored nasal drainage \\
\hline Productive cough & None & Productive cough with yellow sputum \\
\hline Sinus symptoms & None & Complains of sinus pressure and pain \\
\hline Duration of illness & 3-5 days & 14 days \\
\hline Severity of illness & Feels only moderately ill & Feels illness so severe that treatment is needed \\
\hline Temperature & $99 \mathrm{~F}$ & $101.5 \mathrm{~F}$ \\
\hline Expects antibiotics & No specific expectations about treatment & Has come specifically to get antibiotic treatment \\
\hline Pending trip & No trips scheduled & $\begin{array}{l}\text { Leaving on vacation soon, worries about } \\
\text { illness getting worse }\end{array}$ \\
\hline Prior antibiotics & $\begin{array}{l}\text { No prior antibiotics for } \\
\text { this sort of illness }\end{array}$ & $\begin{array}{l}\text { Was previously given antibiotics for similar } \\
\text { illness and had good results }\end{array}$ \\
\hline
\end{tabular}

To reduce the number of cases each participant had to evaluate, the findings are presented using a fractional factorial design that presents all important combinations of findings and allows analysis of the main effects and selected first-order interactions in 20 cases rather than the 512 that would be required for a full factorial [14].

\subsection{Participants}

We recruited emergency physicians from members of the Wisconsin Chapter of the American College of Emergency Physicians (WACEP). Paper case vignettes were sent to all 272 members of the WACEP. We sent a second mailing to those who did not respond. A total of 104 (38\%) emergency physicians returned completed questionnaires. We did not survey other providers, such as nurse practitioners, that might also be working in emergency departments. The survey was conducted in 2006. No demographic data was collected from the participants. The study was reviewed by the Institutional Review Board and considered to be exempt.

\subsection{Analysis}

We derived the weights for the clinical variables using the method of judgment analysis [15] [16]. Participants did not explicitly state whether or not they were influenced by a variable. Rather, a linear model for each practitioner was constructed using regression analysis to infer the weight of each variable from the judgment made about each case (the likelihood they would prescribe antibiotics] given the presence or absence of that variable. We used an idiographic approach: i.e., we first calculated each individual practitioner's weights and then combined the results to produce aggregate data. Analyses were done with the SAS statistical programs (SAS Institute, Inc. version v9.2 Cary, North Carolina). We then compared the results with those of the Colorado primary care practitioners previously reported [14].

\section{Results}

The 104 emergency physicians were more likely to prescribe antibiotics than the Colorado primary care practitioners. The average likelihood of prescribing an antibiotic was 50.4\% (95\% CI 49.0 - 51.8) for our population compared with $43.6 \%$ (95\% C.I 42.1 - 45.1) for the Colorado community practitioners, with a median likelihood of $50 \%$ and $40 \%$ respectively for the 2 groups. Emergency physicians said they would prescribe an antibiotic (yes/no) in $51.4 \%$ of cases for our study, compared with $44.5 \%$ in the Colorado study.

\subsection{Judgment Analysis}

The 104 individual judgment models were first summarized by averaging individual practitioner's weights for each factor. Figure 1 compares the average weighting pattern of the ED physicians to the primary care practitioners. The patterns are strikingly similar. As with the community practitioners, emergency physicians gave by far the most weight to the duration of the illness ( 3 to 5 days versus 14 days). Both groups gave virtually no 


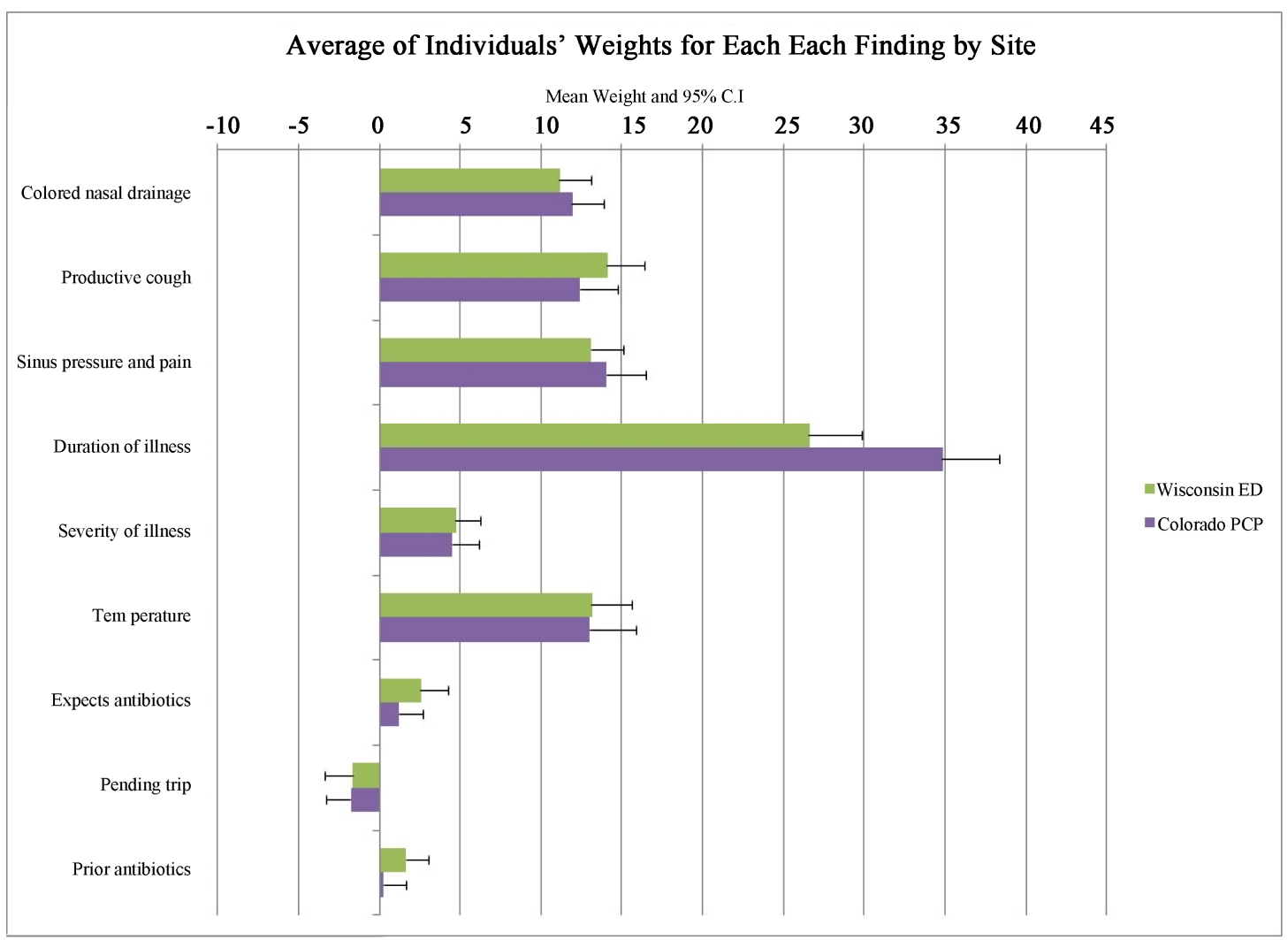

Figure 1. Average of individuals' weights for each factor.

weight to patient expectation for antibiotics, report of prior antibiotic efficacy, or a report of pending travel. Of the remaining factors, severity of illness received the least average weight at about five percent of total.

To determine if the weighting of any factor was influenced by the state of other factors, we calculated the first order interactions. In this study productive cough $\mathrm{x}$ temperature and duration $\mathrm{x}$ temperature were significant, as they had been in the Colorado study [14].

\subsection{Variation among Individual Practitioners}

ED physicians varied considerably in both the patterns of weights of the clinical or patient factors and the range of weights for each individual factor. There was concordance, however, among individual physicians regarding which factor had the greatest weight. Duration of illness was the most important factor for both groups. For each factor, Figure 2 shows the percent of individuals in each group giving that factor the highest weight in the decision to prescribe antibiotics. The results in the emergency physicians were similar to that in the Colorado study both in terms of degree of variability and in the relative weighting of factor importance.

\section{Discussion}

In this judgment analysis of ED physicians' strategies for antibiotic prescribing in patients with ARI, we found a greater propensity to prescribe antibiotics among the ED physicians compared to a previously studied group of community practitioners who made judgments about the identical set of clinical vignettes [14]. Despite the higher likelihood of prescribing antibiotics among the ED physicians, the two groups showed nearly identical aggregate patterns in how clinical and patient factors influenced their decisions. Thus the differences in antibiotic prescribing could not be accounted by differences in how the two groups weighted either the clinical or the patient factors in the vignettes. On average, an ED physician in this study was more likely to recommend antibiotics than a community practitioner evaluating the same vignette.

The high rate of antibiotic recommendations by ED physicians in our study is also reported in actual patient 


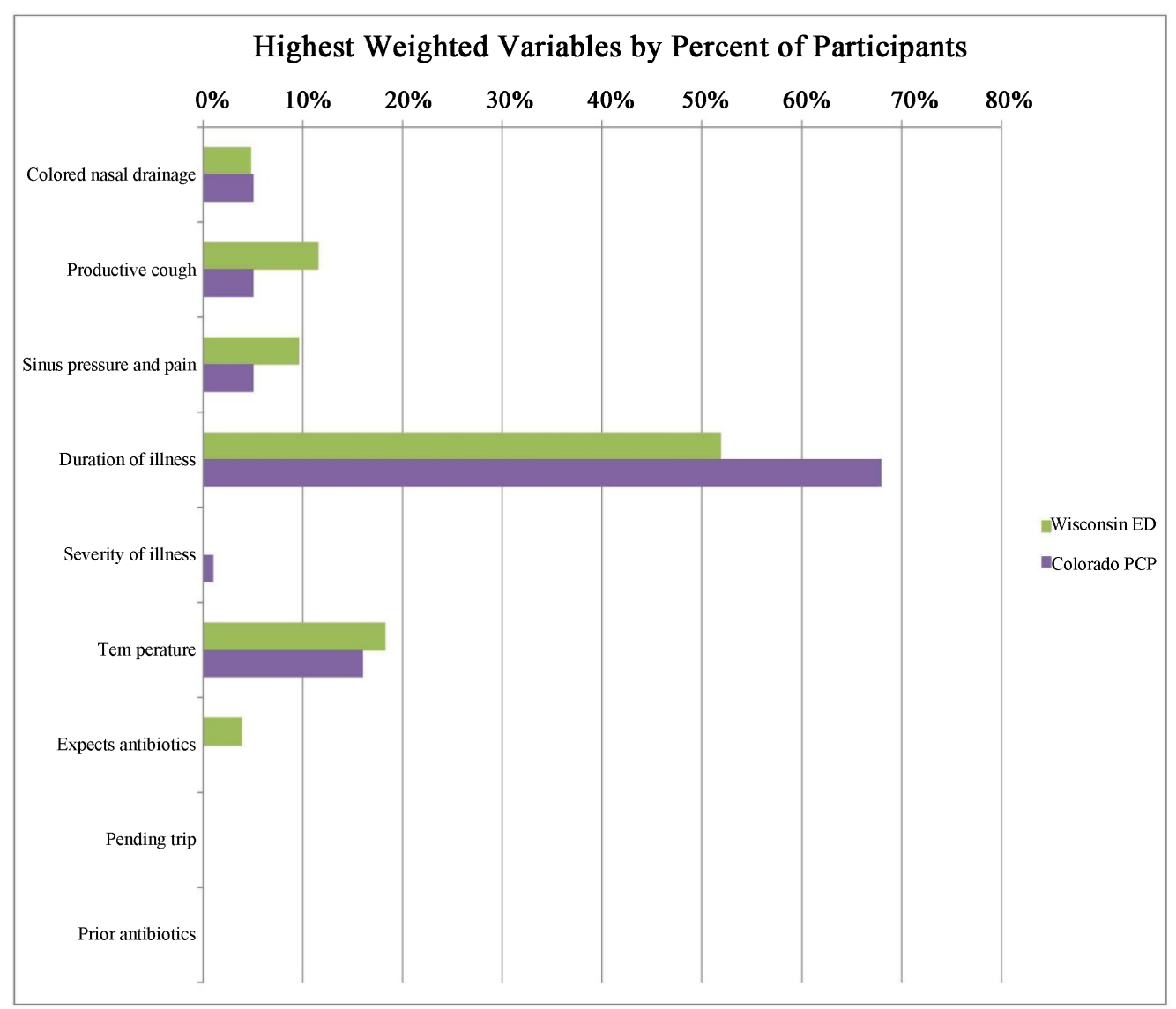

Figure 2. Weights of variables by percentage of participants.

settings. Gonzales et al examined the patterns of antibiotic use for ARIs in acute care settings, which included veterans affairs (VA) and non VA EDs [17]. Of 2270 ARI visits, 62\% were for antibiotic-nonresponsive diagnoses (such as acute bronchitis): $72 \%$ of acute bronchitis and $38 \%$ of URI visits were treated with antibiotics. These data suggest that inappropriate prescribing is a major problem in the ED setting. In reviewing patients diagnosed with bronchitis in ED patients in the Pittsburgh Healthcare System and the Philadelphia VA Medical Center, Aspinal and colleagues found that antibiotics were prescribed in $78 \%$ of patients [18].

In this study, we sought to achieve a better understanding of clinical factors that may influence emergency physicians' decisions about prescribing antibiotics in ARIs. Our results also show that duration of illness is the most important factor influencing prescribing in these cases: the weighting for duration was twice that of any other factor, as it was in the Colorado study as well [14]. Other than for acute rhinosinusitis, where longer duration may make a bacterial etiology more likely, there is little reason to think that a longer duration of illness would warrant more antibiotic use. The interactions of duration with cough and temperature support the hypothesis that over-prescribing for bronchitis is an important contributor.

In our study, we did not find that emergency physicians rated patient expectations or any of the other patient factors as important when considering antibiotic prescribing. This is in contrast to other studies that have found that patient factors are important elements influencing prescribing [11] [13] [19] [20]. In a study of adults and children presenting with symptoms consistent with upper respiratory infection in ten academic EDs, Ong et al. found that physician factors associated with greater likelihood of prescribing antibiotics included patient expectation, although they were able to correctly identify only $27 \%$ of the patients who expected antibiotics [11].

There are several possibilities for the lack of importance of patient factors in the decision to prescribe antibiotics. Since most studies of patient factors rely on physicians' self-report of what influences their decision, it may be that practitioners overestimate the effect of patient factors or may use them to rationalize a decision to prescribe. Previous studies have shown that decision makers often have poor insight into their own decision policies 
and that the weights derived from judgment analysis are better at predicting future decisions [21]. An alternative explanation is that patient factors are indeed important, but that the written descriptions of the patients' wishes in this study did not have the impact of a face-to-face presentation and thus underestimated their effect.

Besides non-clinical patient factors, another possible reason that ED physicians may prescribe more antibiotics for ARI is that they may consider the rate of bacterial etiology of ARIs to be higher in the ED population because they are sicker on presentation. This patient factor was represented in the vignettes by whether the patient "considered the illness so severe that treatment is needed". This factor, however, received the lowest weighting of all clinical factors in deciding on whether to prescribe antibiotics. This lack of influence makes it less likely that the higher prescribing rate by ED physicians is due to an impression that the ED patients are sicker.

Summarizing the findings of our judgment analysis, we cannot account for the ED physicians' higher likelihood to recommend antibiotics based on how they weighted any of the factors in our case vignettes. A possible explanation for this finding would be expectation bias such that ED physicians might expect to see more patients in their practice setting for whom antibiotics are indicated compared with community practitioners. Their perceived base rate of patients needing antibiotics may have influenced their likelihood of recommending antibiotics independently of their perception of the weight of any given clinical factor. This expectation bias across different specialties was demonstrated many years ago in a study of children being evaluated for tonsillectomy where physician characteristics rather than patient characteristics determined whether or not tonsillectomy was recommended by them [22].

Our study has several limitations. The decisions were made in response to paper case vignettes limited to 9 features and not actual patients. Descriptions of clinical findings and patient factors may have lacked the force they would have in patient encounters. Our sample was limited to board certified emergency physicians, and our findings may not be generalizable to urgent care centers or other health care environments that may employ part time MDs, physician assistants, or nurse practitioners. Practice patterns of these Wisconsin practitioners may vary compared with other geographic areas. We did not collect demographic data on the practitioners, such as age, gender, years in practice and type of population they provide care to, and those factors may play a role in prescribing decisions. Finally, the vignettes did not allow ordering further examinations or tests as might have been appropriate if pneumonia had been suspected. Finally, we received responses from $38 \%$ of our sample. However, unlike conventional surveys, the response rate is not as important for the main results in the study since we are not generalizing to the whole population of emergency physicians but instead are analyzing individual behaviors. Even when we compare rates of antibiotic prescriptions across types of providers, we clarify that we are not generalizing to the emergency physician population as a whole (or to the population of community practitioners). We cannot think of a response bias that would significantly affect cue weighting, since people are rarely aware of their own weighting.

In conclusion, we found that these emergency physicians prescribed antibiotics more frequently than PCPs responding to the same paper cases in an earlier study. These rates were both considerably higher than those of physicians who were instructed to apply the CDC guidelines for treatment of ARI. Although the treatment rates were higher among the emergency physicians, their weighting of the clinical findings was very similar to the primary care group, showing an influence of duration of illness double that of any other finding. This result suggests that differences in the importance given to findings are not the source of the difference in prescription rate.

\section{Funding}

None.

\section{Conflict of Interest}

None for all authors.

\section{References}

[1] Gonzales, R., Bartlett, J.G., Besser, R.E., et al. (2001) Principles of Appropriate Antibiotic Use for Treatment of Acute Respiratory Tract Infections in Adults: Background, Specific Aims, and Methods. Annals of Emergency Medicine, 37, 690-697. http://dx.doi.org/10.1067/S0196-0644(01)70087-X 
[2] Hickner, J.M., Bartlett, J.G., Besser, R.E., Gonzales, R., Hoffman, J.R. and Sande, M.A. (2001) Principles of Appropriate Antibiotic Use for Acute Rhinosinusitis in Adults: Background. Annals of Emergency Medicine, 37, 703-710. http://dx.doi.org/10.1067/S0196-0644(01)70089-3

[3] Gonzales, R., Bartlett, J.G., Besser, R.E., Hickner, J.M., Hoffman, J.R. and Sande, M.A. (2001) Principles of Appropriate Antibiotic Use for Treatment of Nonspecific upper Respiratory Tract Infections in Adults: Background. Annals of Emergency Medicine, 134, 490-494. http://dx.doi.org/10.7326/0003-4819-134-6-200103200-00015

[4] Gonzales, R., Bartlett, J.G., Besser, R.E., et al. (2001) Principles of Appropriate Antibiotic Use for Treatment of Uncomplicated Acute Bronchitis: Background. Annals of Emergency Medicine, 37, 720-727. http://dx.doi.org/10.1067/S0196-0644(01)70091-1

[5] Roumie, C.L., Halasa, N.B., Grijalva, C.G., et al. (2005) Trends in Antibiotic Prescribing for Adults in the United States-1995 to 2002. Journal of General Internal Medicine, 20, 697-702. http://dx.doi.org/10.1111/j.1525-1497.2005.0148.x

[6] Gonzales, R., Corbett, K.K., Wong, S., et al. (2008) Get Smart Colorado: Impact of a Mass Media Campaign to Improve Community Antibiotic Use. Medical Care, 46, 597-605. http://dx.doi.org/10.1097/MLR.0b013e3181653d2e

[7] Belongia, E.A., Knobloch, M.J., Kieke, B.A., Davis, J.P., Janette, C. and Besser, R.E. (2005) Impact of Statewide Program to Promote Appropriate Antimicrobial Drug Use. Emerging Infectious Diseases, 11, 912-920.

[8] Vanderweil, S.G., Pelletier, A.J., Hamedani, A.G., Gonzales, R., Metlay, J.P. and Camargo, Jr., C.A. (2007) Declining Antibiotic Prescriptions for Upper Respiratory Infections, 1993-2004. Academic Emergency Medicine, 14, 366-369.

[9] Thorpe, J.M., Smith, S.R. and Trygstad, T.K. (2004) Trends in Emergency Department Antibiotic Prescribing for Acute Respiratory Tract Infections. Annals of Pharmacotherapy, 38, 928-935. http://dx.doi.org/10.1345/aph.1D380

[10] Gonzales, R., Camargo, Jr., C.A., MacKenzie, T., et al. (2006) Antibiotic Treatment of Acute Respiratory Infections in Acute Care Settings. Academic Emergency Medicine, 13, 288-294. http://dx.doi.org/10.1111/j.1553-2712.2006.tb01695.x

[11] Ong, S., Nakase, J., Moran, G.J., Karras, D.J., Kuehnert, M.J. and Talan, D.A. (2007) Antibiotic Use for Emergency Department Patients with Upper Respiratory Infections: Prescribing Practices, Patient Expectations, and Patient Satisfaction. Annals of Emergency Medicine, 50, 213-220. http://dx.doi.org/10.1016/j.annemergmed.2007.03.026

[12] Gonzales, R., Barrett, Jr., P.H., Crane, L.A. and Steiner, J.F. (1998) Factors Associated with Antibiotic Use for Acute Bronchitis. Journal of General Internal Medicine, 13, 541-548. http://dx.doi.org/10.1046/j.1525-1497.1998.00165.x

[13] Mangione-Smith, R., McGlynn, E.A., Elliott, M.N., Krogstad, P. and Brook, R.H. (1999) The Relationship between Perceived Parental Expectations and Pediatrician Antimicrobial Prescribing Behavior. Pediatrics, 103, 711-718. http://dx.doi.org/10.1542/peds.103.4.711

[14] Wigton, R.S., Darr, C.A., Corbett, K.K., Nickol, D.R. and Gonzales, R. (2008) How Do Community Practitioners Decide Whether to Prescribe Antibiotics for Acute Respiratory Tract Infections? Journal of General Internal Medicine, 23, 1615-1620. http://dx.doi.org/10.1007/s11606-008-0707-9

[15] Cooksey, R.W. (1996) Judgement Analysis: Theory, Methods and Applications. Academic Press, Waltham.

[16] Wigton, R.S. (1988) Use of Linear Models to Analyze Physicians’ Decisions. Medical Decision Making, 8, $241-252$. http://dx.doi.org/10.1177/0272989X8800800404

[17] Gonzales, R., Bartlett, J.G., Besser, R.E., Cooper, R.J., Hickner, J.M., Hoffman, J.R., et al. (2001) Principles of Appropriate Antibiotic Use for Treatment of Acute Respiratory Tract Infections in Adults: Background, Specific Aims, and Methods. Annals of Internal Medicine, 134, 479-486. http://dx.doi.org/10.7326/0003-4819-134-6-200103200-00013

[18] Aspinall, S.L., Good, C.B., Metlay, J.P., Mor, M.K. and Fine, M.J. (2009) Antibiotic Prescribing for Presumed Nonbactrial Acute Respiratory Tract Infections. Annals of Emergency Medicine, 27, 544-551.

[19] Stearns, C.R., Gonzales, R., Camargo, Jr., C.A., Maselli, J. and Metlay, J.P. (2009) Antibiotic Prescriptions Are Associated with Increased Patient Satisfaction with Emergency Department Visits for Acute Respiratory Tract Infections. Academic Emergency Medicine, 16, 934-941. http://dx.doi.org/10.1111/j.1553-2712.2009.00522.x

[20] Belongia, E.A., Naimi, T.S., Gale, C.M. and Besser, R.E. (2002) Antibiotic Use and Upper Respiratory Infections: A Survey of Knowledge, Attitudes, and Experience in Wisconsin and Minnesota. Preventive Medicine, 34, 346-352. http://dx.doi.org/10.1006/pmed.2001.0992

[21] Kirwan, J.R., Chaput de Saintonge, D.M., Joyce, C.R., Holmes, J. and Currey, H.L. (1986) Inability of Rheumatologists to Describe Their True Policies for Assessing Rheumatoid Arthritis. Annals of the Rheumatic Diseases, 45, 156161. http://dx.doi.org/10.1136/ard.45.2.156

[22] Bakwin, H. (1946) Pseudodoxia Pediatrica. The New England Journal of Medicine, 232, 691-697. http://dx.doi.org/10.1056/NEJM194506142322401 